Pedro Ángel Sangucho-Verdezoto; Marcos Edisson Bustillos-Solórzano; Ana Abigail López-Guerrero; Augusta Nataly Guerra-Zambrano

http://dx.doi.org/10.35381/s.v.v4i8.1001

\title{
Aspectos sociodemográficos y desarrollo de complicaciones en el período de gestación
}

\section{Sociodemographic aspects and development of complications in the gestation period}

Pedro Ángel Sangucho-Verdezoto

psangucho9720@uta.edu.ec

Postgrado de Medicina Familiar y Comunitaria, Facultad de Ciencias de la Salud, Universidad Técnica de Ambato, Ambato

Ecuador

https://orcid.org/0000-0003-0255-8609

Marcos Edisson Bustillos-Solórzano

marcoseddybs@gmail.com

Postgrado de Medicina Familiar y Comunitaria, Facultad de Ciencias de la Salud, Universidad Técnica de Ambato, Ambato

Ecuador

https://orcid.org/0000-0001-6310-530X

Ana Abigail López-Guerrero

anbilo18@hotmail.com

Postgrado de Medicina Familiar y Comunitaria, Facultad de Ciencias de la Salud, Universidad Técnica de Ambato, Ambato

Ecuador

https://orcid.org/0000-0002-3565-4296

Augusta Nataly Guerra-Zambrano

anathy1986@hotmail.com

Postgrado de Medicina Familiar y Comunitaria, Facultad de Ciencias de la Salud, Universidad Técnica de Ambato, Ambato

Ecuador

https://orcid.org/0000-0001-9207-9235

Recepción: 15 de junio 2020

Revisado: 28 de julio 2020

Aprobación: 25 de agosto 2020

Publicación: 03 de septiembre 2020 
Pedro Ángel Sangucho-Verdezoto; Marcos Edisson Bustillos-Solórzano; Ana Abigail López-Guerrero; Augusta Nataly Guerra-Zambrano

\title{
RESUMEN
}

Objetivo: Establecer los aspectos sociodemográficos y el desarrollo de complicaciones en el período de gestación en una comunidad ecuatoriana. Método: La investigación se realizó a través de un estudio descriptivo, prospectivo, de corte transversal, mediante un seguimiento de 12 meses del comportamiento de la gestación de cada una de las pacientes que cumplieron con los criterios de inclusión. Resultados: se pudo establecer que alrededor del $25 \%$ de los casos si existía el riesgo de presentar por lo menos una complicación a lo largo del embarazo, en la finalización del mismo y en el puerperio inmediato. Conclusión: Estos factores de riesgo que afectaron tanto a la madre como al producto de la concepción, estaban relacionados con el nivel educativo, edad, estado civil de cada paciente y su entorno.

Descriptores: Embarazo; preeclampsia; mujeres. (Fuente DeCS, 2020).

\begin{abstract}
Objective: To establish the sociodemographic aspects and the development of complications in the gestation period in an Ecuadorian community. Methods: The research was carried out through a descriptive, prospective, cross-sectional study by means of a 12-month follow-up of the gestation behavior of each one of the patients who met the inclusion criteria. Results: It could be established that about $25 \%$ of the cases if there was a risk of presenting at least one complication during pregnancy, at the end of it and in the immediate postpartum period. Conclusion: These risk factors that affected both the mother and the product of conception were related to the educational level, age, marital status of each patient and their environment.
\end{abstract}

Descriptors: Pregnancy; pre-eclampsia; women. (Source DeCS, 2020). 
Pedro Ángel Sangucho-Verdezoto; Marcos Edisson Bustillos-Solórzano; Ana Abigail López-Guerrero; Augusta Nataly Guerra-Zambrano

\section{INTRODUCCIÓN}

El embarazo es un proceso cuyo desarrollo apropiado depende no sólo de los aspectos relacionados con una salud saludable de la madre antes, durante y después de la culminación del mismo; sino que además en esta etapa también influyen las características sociodemográficas de la gestante y su entorno ${ }^{1}$. Esta realidad se puede constatar en los países en vías de desarrollo, en donde el aspecto social en el que se desenvuelve su población influye de manera importante en la aparición de complicaciones durante el período de gestación, situación que se convierte en un reto para ser contrarrestada por los diversos sistemas de salud ${ }^{2}$. La bibliografía relacionada con estos aspectos es limitada en cuanto a número de estudios, actualización y nivel de evidencia válida. 3 realizaron un estudio donde relacionaron los aspectos sociodemográficos con el desarrollo de complicaciones durante el embarazo, los resultados hallados indicaron que los aspectos sociodemográficos influyen de manera significativa en la aparición de complicaciones durante la etapa de gestación.

El Ecuador gracias a la implementación del nuevo Modelo de atención integral de Salud, ha tratado de implementar al sistema nacional de salud la parte integral en la valoración de salud. ${ }^{4}$ Por lo tanto, el análisis de una determinada morbilidad se la realiza desde el punto de vista biológico, asociándose con el entorno psicosocial de cada individuo, esta es la verdadera esencia del Médico Familiar que se desenvuelve en el nivel primario de Salud 5 .

Las consideraciones de la asociación existente entre las determinantes demográficas con los aspectos biológicos de una mujer gestante fueron descritas por ${ }^{6}$, mediante una investigación en la que se describió la relación entre la edad, nivel educativo y económico maternos como factores de riesgo para la presencia de complicaciones en el embarazo.

Las variables descritas con anterioridad además influyen en el estilo de vida de la mujer embarazada, el cual cuando se relaciona con aspectos que alteran el índice de masa 
Pedro Ángel Sangucho-Verdezoto; Marcos Edisson Bustillos-Solórzano; Ana Abigail López-Guerrero;

corporal, representan un riesgo para la consecución de morbilidades durante el periodo de gestación ${ }^{7}$.

El objetivo de la investigación se basó en establecer los aspectos sociodemográficos y el desarrollo de complicaciones en el período de gestación en una comunidad ecuatoriana.

\section{MATERIAL Y MÉTODOS}

\section{Tipo y diseño de investigación}

La investigación fue de tipo prospectivo, descriptiva observacional con una duración de 12 meses, en los cuales se realizó la selección y análisis minucioso de la información completa contenida en los archivos clínicos de cada gestante, respetando el principio de la confidencialidad, proceso realizado en los 5 controles prenatales de cada gestante.

\section{Muestra poblacional}

La población estuvo conformada por las mujeres que acudieron a sus controles prenatales a la unidad de salud tipo $\mathrm{C}$, ubicada en la ciudad de Latacunga-Ecuador. En el primer control se captaron un total de 96 pacientes, aquí se establecieron los mecanismos y directrices necesarias para identificar los factores de riesgo que asociados a las variables sociodemográficas desencadenen complicaciones a lo largo del progreso del embarazo.

En el segundo control prenatal se identificaron 27 casos de complicaciones relacionadas principalmente con infecciones del tracto genitourinario, anemia y amenaza de aborto. El tercer control con 19 casos de gestantes con complicaciones; el cuarto control con 23 casos asociados a la adolescencia, mujeres añosas y menor grado de instrucción educativa. En el quinto con 24 casos de morbilidades. 
Pedro Ángel Sangucho-Verdezoto; Marcos Edisson Bustillos-Solórzano; Ana Abigail López-Guerrero;

\section{Recopilación de información}

Se recopiló mediante seguimiento y registro clínico del paciente que acudían al centro asistencial, extrayéndose los datos referidos al tema de investigación para su posterior procesamiento estadístico.

\section{Análisis de la información}

El riesgo relativo fue el método estadístico más apropiado para el análisis de la información obtenida ${ }^{8}$.

\section{RESULTADOS}

\section{Tabla 1.}

Complicaciones en el segundo control según las variables demográficas.

\begin{tabular}{|c|c|c|c|c|c|}
\hline Edad & $\begin{array}{c}\text { Riesgo } \\
\text { relativo }\end{array}$ & Instrucción & $\begin{array}{c}\text { Riesgo } \\
\text { relativo }\end{array}$ & $\begin{array}{c}\text { Estado } \\
\text { civil }\end{array}$ & $\begin{array}{c}\text { Riesgo } \\
\text { relativo }\end{array}$ \\
\hline Adolescentes & 1,1 & Primaria & 0,47 & Soltera & 0,99 \\
\hline Adultas & 1,24 & Secundaria & 1,1 & Casada & 0,77 \\
\hline Madre añosa & 1,19 & Superior & 1,23 & $\begin{array}{c}\text { Unión } \\
\text { libre }\end{array}$ & 1,44 \\
\hline
\end{tabular}

Fuente: Archivos clínicos. Elaborado por los autores.

Los datos obtenidos en el segundo control demuestran que, de las tres variables demográficas estudiadas, la edad en los tres grupos analizados si tuvieron más probabilidad de presentar alguna complicación del embarazo, (RR 1,1-1,24-1,29 respectivamente) El nivel de instrucción influyó en 2 de los 3 grupos analizados (RR 1,11,23 respectivamente). Por último, en lo referente al estado civil, engrupo en unión libre tuvieron 1,44 más probabilidades de presentar una determinada complicación en el 
Pedro Ángel Sangucho-Verdezoto; Marcos Edisson Bustillos-Solórzano; Ana Abigail López-Guerrero;

embarazo (tabla 1).

Tabla 2.

Complicaciones en el tercer control según las variables demográficas.

\begin{tabular}{|c|c|c|c|c|c|}
\hline Edad & $\begin{array}{c}\text { Riesgo } \\
\text { relativo }\end{array}$ & Instrucción & $\begin{array}{c}\text { Riesgo } \\
\text { relativo }\end{array}$ & $\begin{array}{c}\text { Estado } \\
\text { civil }\end{array}$ & $\begin{array}{c}\text { Riesgo } \\
\text { relativo }\end{array}$ \\
\hline Adolescentes & 1,54 & Primaria & 1,08 & Soltera & 0,81 \\
\hline Adultas & 0,61 & Secundaria & 0,96 & Casada & 7,91 \\
\hline Madre añosa & 1,7 & Superior & 1 & Unión libre & 0,11 \\
\hline
\end{tabular}

Fuente: Elaborado por los autores. Archivos clínicos.

En el tercer control las adolescentes y madres añosas, tuvieron más probabilidades de presentar alguna complicación ( $R R$ 1,54 y 1,7) respectivamente. En lo referente al grado de instrucción y estado civil, aquellas gestantes con primaria y casadas tuvieron mayor probabilidad (RR1,08 y 7,91) (tabla 2).

Tabla 3.

Complicaciones en el cuarto control según las variables demográficas.

\begin{tabular}{|c|c|c|c|c|c|}
\hline Edad & $\begin{array}{c}\text { Riesgo } \\
\text { relativo }\end{array}$ & Instrucción & $\begin{array}{c}\text { Riesgo } \\
\text { relativo }\end{array}$ & $\begin{array}{c}\text { Estado } \\
\text { civil }\end{array}$ & $\begin{array}{c}\text { Riesgo } \\
\text { relativo }\end{array}$ \\
\hline Adolescentes & 3,09 & Primaria & 2,04 & Soltera & 1,4 \\
\hline Adultas & 0,28 & Secundaria & 0,66 & Casada & 4,76 \\
\hline Madre añosa & 2,92 & Superior & 0,95 & Unión libre & 0,2 \\
\hline
\end{tabular}

Fuente: Elaborado por los autores. Archivos clínicos. 
Pedro Ángel Sangucho-Verdezoto; Marcos Edisson Bustillos-Solórzano; Ana Abigail López-Guerrero;

En esta etapa del embarazo, el cuarto control, se repitieron los resultados en lo referente a edad e instrucción (RR 3,09-2,92 y 2,04) respectivamente. En la variable estado civil las soteras y casadas presentaron mayor probabilidad (RR 1,4 y 4,76$)$ (tabla 3 ).

\section{Tabla 4.}

Complicaciones en el quinto control según las variables demográficas.

\begin{tabular}{|c|c|c|c|c|c|}
\hline Edad & $\begin{array}{c}\text { Riesgo } \\
\text { relativo }\end{array}$ & Instrucción & $\begin{array}{c}\text { Riesgo } \\
\text { relativo }\end{array}$ & $\begin{array}{c}\text { Estado } \\
\text { civil }\end{array}$ & $\begin{array}{c}\text { Riesgo } \\
\text { relativo }\end{array}$ \\
\hline Adolescentes & 3,47 & Primaria & 2,38 & Soltera & 0,9 \\
\hline Adultas & 0,26 & Secundaria & 0,73 & Casada & 3,63 \\
\hline Madre añosa & 2,92 & Superior & 0,72 & Unión libre & 0,69 \\
\hline
\end{tabular}

Fuente: Elaborado por los autores. Archivos clínicos.

En el quinto control se mantuvo la tendencia hallada en las citas anteriores para la edad (RR 3,47 y 2,92) e instrucción (RR 2,38) respectivamente. En el caso del estado civil, en las casadas existió mayor probabilidad (RR 3,63).
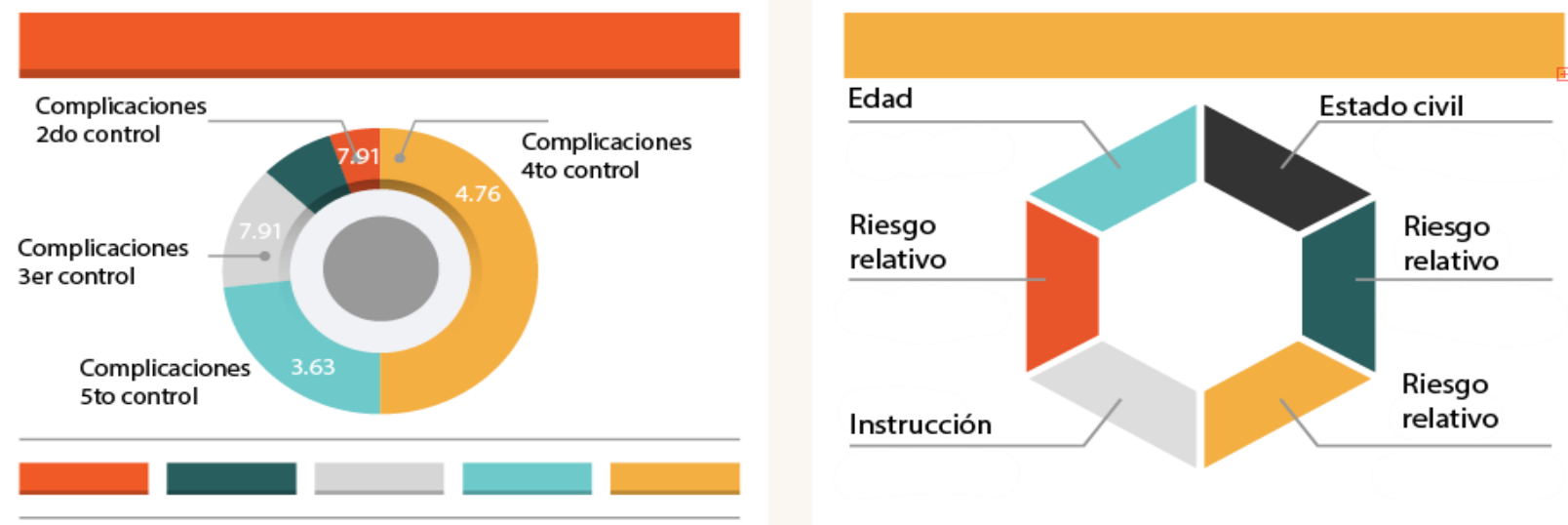

Figura 1. Análisis de los resultados obtenidos en los archivos clínicos. 
Pedro Ángel Sangucho-Verdezoto; Marcos Edisson Bustillos-Solórzano; Ana Abigail López-Guerrero; Augusta Nataly Guerra-Zambrano

\section{DISCUSIÓN}

La edad influyó en el riesgo para el desarrollo de complicaciones en la gestación, característica que se presentó en todos los controles de la evolución del embarazo en esta investigación. Los resultados fueron similares a los obtenidos por ${ }^{9}$, estudio en el cual además hallaron la relación de la presencia de morbilidad materna asociada con el nivel educativo y estado civil de la gestante.

Varios estudios arrojan información donde implican a la adolescencia materna como un factor de riesgo para desarrollar complicaciones ${ }^{10}$. Sin embargo, demostraron que existe una tendencia similar en las mujeres añosas ${ }^{11}$. En nuestro estudio ambas variables si representaron un aumento en la probabilidad de morbilidad en el embarazo en todos los controles médico.

El nivel educativo de la madre influye en el ámbito de la cultura y estilo de vida de un individuo, de manera que mientras menor sea la educación las probabilidades de presentar complicaciones en el embarazo se incrementan ${ }^{12}$. Los hallazgos fueron similares en nuestra investigación.

El estado civil también tiene una tendencia a influir en la probabilidad de crear una complicación en el período de gestación ${ }^{13}$. Por lo tanto, el ámbito social en el que esta inmiscuido un individuo influye en el desarrollo de enfermedades ${ }^{14}$. En este estudio la probabilidad fue mayor en las mujeres casadas en todos los controles.

La visión integral por lo tanto resulta importante, para impartir medidas encaminadas a la prevención del desarrollo de enfermedades ${ }^{15}$. En este aspecto juega un papel decisivo el Médico de Familia.

\section{CONFLICTO DE INTERÉS}

Los autores declaran que no tienen conflicto de interés en la publicación de este artículo. 
Pedro Ángel Sangucho-Verdezoto; Marcos Edisson Bustillos-Solórzano; Ana Abigail López-Guerrero; Augusta Nataly Guerra-Zambrano

\section{FINANCIAMIENTO}

Autofinanciado.

\section{AGRADECIMIENTO}

Al Centro de posgrado de la Universidad Técnica de Ambato. Especialización en Medicina Familiar y Comunitaria, segunda cohorte; por el apoyo en el desarrollo de la investigación.

\section{REFERENCIAS}

1. Goldstein RF, Abell SK, Ranasinha S, Misso ML, Boyle JA, Harrison CL, Black MH, Li N, Hu G, Corrado F, Hegaard H, Kim YJ, Haugen M, Song WO, Kim MH, Bogaerts A, Devlieger R, Chung JH, Teede HJ. Gestational weight gain across continents and ethnicity: systematic review and meta-analysis of maternal and infant outcomes in more than one million women. BMC Med. 2018 Aug 31;16 (1):153. doi: 10.1186/s12916-018-1128-1. PMID: 30165842; PMCID: PMC6117916.

2 Ahmad N, Nor SFS, Daud F. Understanding Myths in Pregnancy and Childbirth and the Potential Adverse Consequences: A Systematic Review. Malays J Med Sci. 2019 Jul;26(4):17-27. doi: 10.21315/mjms2019.26.4.3. Epub 2019 Aug 29. PMID: 31496890; PMCID: PMC6719884.

3. Jiménez MA, Martín AR, García JR. Comparing the biological and psychosocial risks of pregnancy between groups of adolescents and adults. Eur $\mathrm{J}$ Epidemiol. 2000 Jun;16(6):527-32. doi: 10.1023/a:1007680005731. PMID: 11049096.

4. Naranjo-Ferregut J, Delgado-Cruz A, Rodríguez-Cruz Roberto, Sánchez-Pérez Y. Consideraciones sobre el Modelo de Atención Integral de Salud del Ecuador [Considerations on the Model of Comprehensive Health Care in Ecuador]. Rev Cubana Med Gen Integr [Internet]. 2014; 30(3). 
Pedro Ángel Sangucho-Verdezoto; Marcos Edisson Bustillos-Solórzano; Ana Abigail López-Guerrero; Augusta Nataly Guerra-Zambrano

5. Dube YP, Nyapwere N, Magee LA, Vidler M, Moore SE, Barratt B, Craik R, von Dadelszen P, Makanga PT, The Precise Network. Interactions between the Physical and Social Environments with Adverse Pregnancy Events Related to Placental Disorders-A Scoping Review. Int J Environ Res Public Health. 2020 Jul 28; 17(15):5421. doi: 10.3390/ijerph17155421. PMID: 32731379; PMCID: PMC7432342.

6. Pinheiro $\mathrm{RL}$, Areia $\mathrm{AL}$, Mota Pinto A, Donato $\mathrm{H}$. Advanced Maternal Age: Adverse Outcomes of Pregnancy, A Meta-Analysis. Acta Med Port. 2019 Mar 29;32(3):219-226. doi: 10.20344/amp.11057. Epub 2019 Mar 29. PMID: 30946794.

7. Aşcı Ö, Rathfisch G. Effect of lifestyle interventions of pregnant women on their dietary habits, lifestyle behaviors, and weight gain: a randomized controlled trial. J Health Popul Nutr. 2016 Feb 24; 35:7. doi: 10.1186/s41043-016-0044-2. PMID: 26911204; PMCID: PMC5025976.

8. Sutradhar R, Austin PC. Relative rates not relative risks: addressing a widespread misinterpretation of hazard ratios. Ann Epidemiol. 2018 Jan;28(1):54-57. doi: 10.1016/j.annepidem.2017.10.014. Epub 2017 Nov 7. PMID: 29239842.

9. Lagadec N, Steinecker M, Kapassi A, Magnier AM, Chastang J, Robert S, Gaouaou N, Ibanez G. Factors influencing the quality of life of pregnant women: a systematic review. BMC Pregnancy Childbirth. 2018 Nov 23;18(1):455. doi: 10.1186/s12884-018-2087-4. PMID: 30470200; PMCID: PMC6251086.

10. Neal S, Mahendra S, Bose K, Camacho AV, Mathai M, Nove A, Santana F, Matthews $Z$. The causes of maternal mortality in adolescents in low and middle income countries: a systematic review of the literature. BMC Pregnancy Childbirth. 2016 Nov 11;16(1):352. doi: 10.1186/s12884-016-1120-8. PMID: 27836005; PMCID: PMC5106816.

11. Leader J, Bajwa A, Lanes A, Hua X, Rennicks White R, Rybak N, Walker M. The Effect of Very Advanced Maternal Age on Maternal and Neonatal Outcomes: A Systematic Review. J Obstet Gynaecol Can. 2018 Sep;40(9):1208-1218. doi: 10.1016/j.jogc.2017.10.027. Epub 2018 Apr 19. PMID: 29681506. 
Pedro Ángel Sangucho-Verdezoto; Marcos Edisson Bustillos-Solórzano; Ana Abigail López-Guerrero;

12. Ketema DB, Leshargie CT, Kibret GD, Assemie MA, Petrucka P, Alebel A. Effects of maternal education on birth preparedness and complication readiness among Ethiopian pregnant women: a systematic review and meta-analysis. BMC Pregnancy Childbirth. 2020 Mar 6;20(1):149. doi: 10.1186/s12884-020-2812-7. PMID: 32143581; PMCID: PMC7060625.

13. Sumankuuro J, Crockett J, Wang S. Sociocultural barriers to maternity services delivery: a qualitative meta-synthesis of the literature. Public Health. 2018 Apr;157:77-85. doi: 10.1016/j.puhe.2018.01.014. Epub 2018 Mar 20. PMID: 29501985.

14. Hadfield $\mathrm{H}$, Wittkowski A. Women's Experiences of Seeking and Receiving Psychological and Psychosocial Interventions for Postpartum Depression: A Systematic Review and Thematic Synthesis of the Qualitative Literature. J Midwifery Womens Health. 2017 Nov;62(6):723-736. doi: 10.1111/jmwh.12669. Epub 2017 Dec 6. PMID: 29210501.

15. Atrash $\mathrm{H}$, Jack B. Preconception care to improve pregnancy outcomes: clinical practice guidelines. J Hum Growth Dev. 2020; 30(3):407-416. DOI: http:doi.org/10.7322/jhgd.v30.11075

(C2020 por los autores. Este artículo es de acceso abierto y distribuido según los términos y condiciones de la licencia Creative Commons Atribución-NoComercial-Compartirlgual 4.0 Internacional (CC BY-NC-SA 4.0) (https://creativecommons.org/licenses/by-nc-sa/4.0/). 